Acta Crystallographica Section E

Structure Reports

Online

ISSN 1600-5368

\section{Malcolm J. Todd and William T. A. Harrison*}

Department of Chemistry, University of Aberdeen, Meston Walk, Aberdeen AB24 3UE, Scotland

Correspondence e-mail:

w.harrison@abdn.ac.uk

\section{Key indicators}

Single-crystal X-ray study

$T=120 \mathrm{~K}$

Mean $\sigma(\mathrm{C}-\mathrm{C})=0.003 \AA$

$R$ factor $=0.030$

$w R$ factor $=0.068$

Data-to-parameter ratio $=19.8$

For details of how these key indicators were automatically derived from the article, see http://journals.iucr.org/e.

\title{
Propane-1,2-diaminium selenite monohydrate
}

The title compound, $\mathrm{C}_{3} \mathrm{H}_{12} \mathrm{~N}_{2}{ }^{2+} \cdot \mathrm{SeO}_{3}{ }^{2-} \cdot \mathrm{H}_{2} \mathrm{O}$, contains a network of propane-1,2-diaminium $\left(\mathrm{C}_{3} \mathrm{H}_{12} \mathrm{~N}_{2}{ }^{2+}\right)$ cations, selenite $\left(\mathrm{SeO}_{3}{ }^{2-}\right)$ anions and water molecules. The crystal packing involves $\mathrm{N}-\mathrm{H} \cdots \mathrm{O}$ [average $\mathrm{H} \cdots \mathrm{O}=1.89 \AA$, $\mathrm{N}-$ $\mathrm{H} \cdots \mathrm{O}=165^{\circ}$ and $\left.\mathrm{N} \cdots \mathrm{O}=2.777(3) \AA\right]$ and $\mathrm{O}-\mathrm{H} \cdots \mathrm{O}$ hydrogen bonds, resulting in a layered structure.

\section{Comment}

The title compound, (I), was prepared as part of our ongoing studies of hydrogen-bonding interactions in the crystal structures of (protonated) amine phosphates (Demir et al., 2003), phosphites (Harrison, 2003), selenites (Ritchie \& Harrison, 2003) and arsenates (Lee \& Harrison, 2003a,b,c; Wilkinson \& Harrison, 2004).<smiles>[NH3+]C[C@H]([NH3+])C[18OH]</smiles>

(I)

The asymmetric unit of (I) contains one $\mathrm{C}_{3} \mathrm{H}_{12} \mathrm{~N}_{2}{ }^{2+}$ cation, one $\mathrm{SeO}_{2}{ }^{2-}$ anion and a water molecule (Fig. 1). The geometric parameters for the organic cation are unexceptional (Lee \& Harrison, 2003a). This species is chiral (C1 has $S$ configuration in the selected asymmetric unit), but crystal symmetry generates a 50:50 mix of enantiomers, consistent

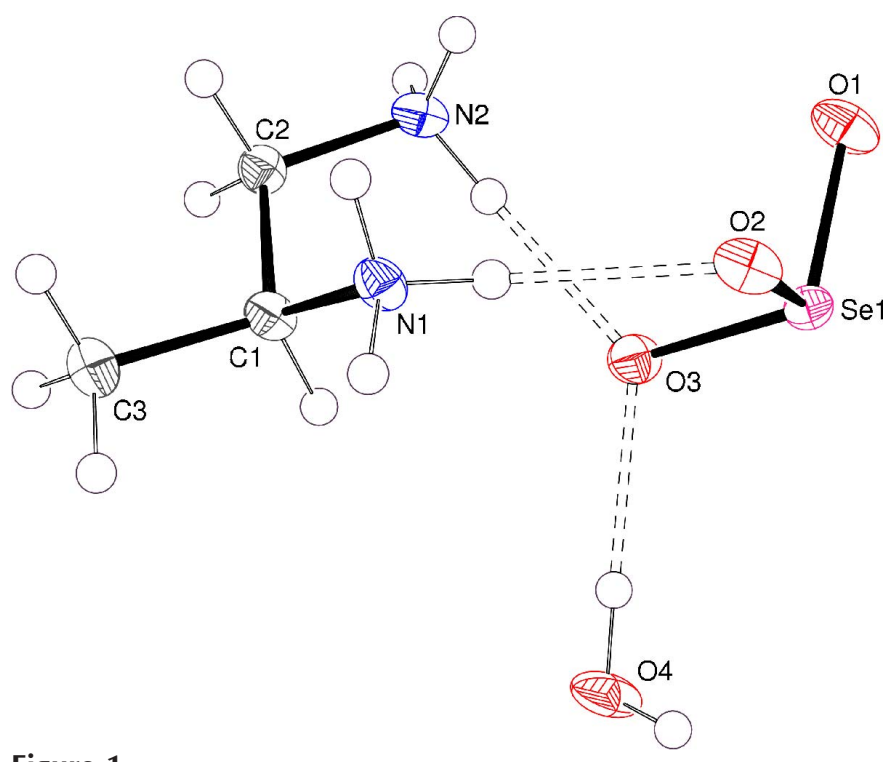

Figure 1

Asymmetric unit of (I), showing 50\% displacement ellipsoids (arbitrary spheres for $\mathrm{H}$ atoms). Hydrogen bonds are indicated by dashed lines.
Received 15 April 2005 Accepted 22 April 2005 Online 7 May 2005
(C) 2005 International Union of Crystallography Printed in Great Britain - all rights reserved 


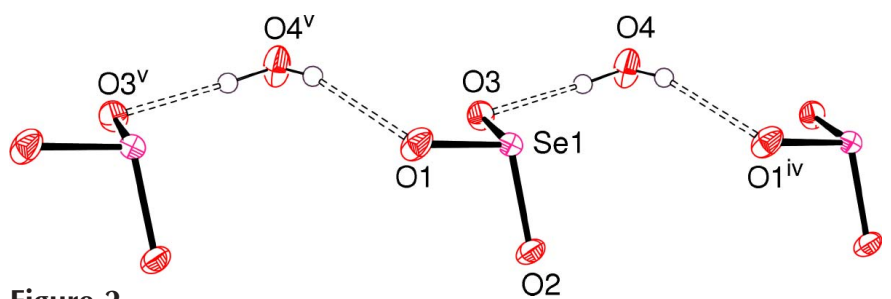

Figure 2

Detail of a hydrogen-bonded selenite/water chain in (I). Symmetry codes are as in Table 2 [additionally: (v) $x, 1+y, z$ ]. Hydrogen bonds are indicated by dashed lines.

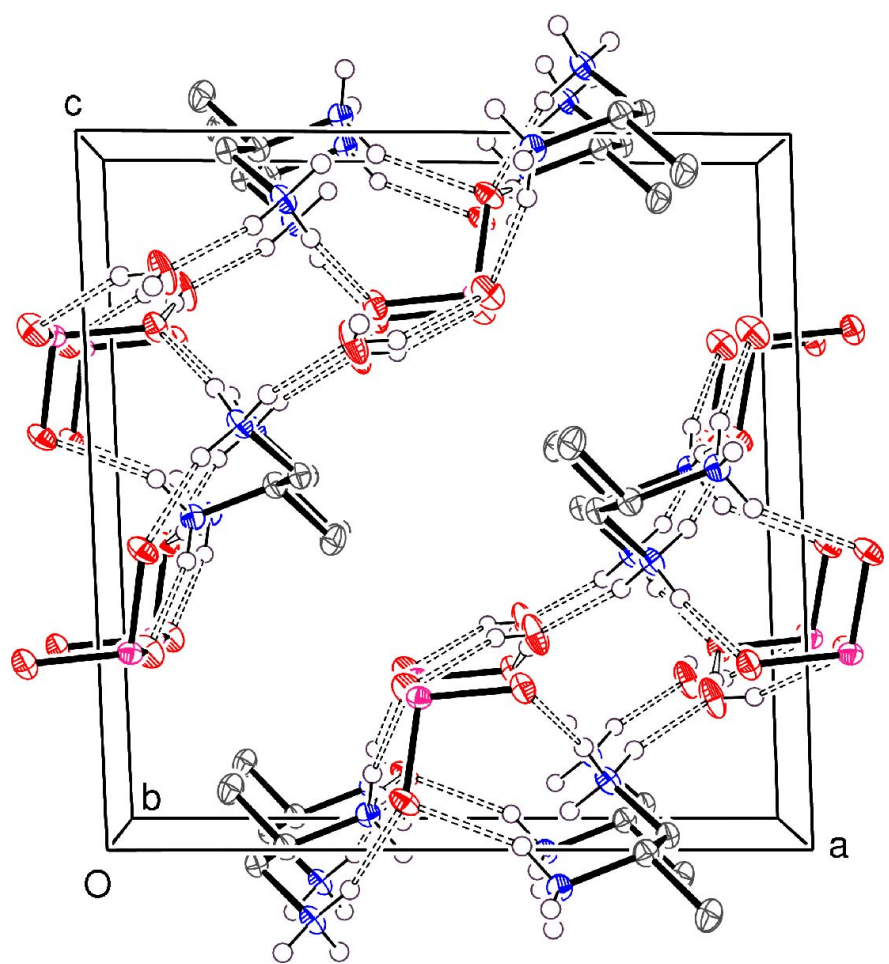

Figure 3

[010] projection of the packing for (I). C-bound $\mathrm{H}$ atoms have been omitted for clarity and hydrogen bonds are indicated by dashed lines.

with the racemic starting material. The selenite group in (I) shows its standard (Lee \& Harrison, 2003) pyramidal geometry (Table 1) [average $\mathrm{Se}-\mathrm{O}=1.687$ (2) $\AA$ ], with the $\mathrm{Se}$ atom displaced from the plane of its three attached $\mathrm{O}$ atoms by 0.7213 (12) $\AA$.

As well as electrostatic attractions, the component species in (I) interact by means of a network of $\mathrm{N}-\mathrm{H} \cdots \mathrm{O}$ and $\mathrm{O}-$ $\mathrm{H} \cdots \mathrm{O}$ hydrogen bonds (Table 2). The selenite anions and water molecules are linked into a polymeric chain in the [010] direction by hydrogen bonds (Fig. 2). The organic species interacts with the selenite/water chains by way of $\mathrm{N}-\mathrm{H} \cdots \mathrm{O}$ hydrogen bonds (Table 2). All six of the $-\mathrm{NH}_{3}{ }^{+} \mathrm{H}$ atoms are involved in these links [mean $\mathrm{H} \cdots \mathrm{O}=1.89 \AA$ 政 $\mathrm{N}-\mathrm{H} \cdots \mathrm{O}=$ $165^{\circ}$ and $\mathrm{N} \cdots \mathrm{O}=2.777$ (3) $\AA$ ]. These interactions result in (101) selenite/water/aminium layers sandwiched between the carbon backbones of the organic groups (Fig. 3), which themselves interact by way of van der Waals forces.

Propane-1,2-diaminium hydrogenarsenate monohydrate, $\mathrm{C}_{3} \mathrm{H}_{12} \mathrm{~N}_{2}{ }^{2+} \cdot \mathrm{HAsO}_{4}{ }^{2-} \cdot \mathrm{H}_{2} \mathrm{O}$ (Lee \& Harrison, 2003a), has an equivalent stoichiometry to (I). As might be expected, where the oxo anion has hydrogen-bonding capability (i.e. as As$\mathrm{OH}$... O links), a quite different overall structure arises. An interesting difference also arises for the organic cation; in (I), the $-\mathrm{NH}_{3}{ }^{+}$and $-\mathrm{CH}_{3}$ groups are trans about their linking $\mathrm{C}-\mathrm{C}$ bond (Table 1), whereas in the hydrogenarsenate, they are gauche $\left[\mathrm{C}-\mathrm{C}-\mathrm{C}-\mathrm{N}=-54.09(18)^{\circ}\right]$.

\section{Experimental}

An aqueous $0.5 \mathrm{M}$ propane-1,2-diamine solution $(10 \mathrm{ml})$ was added to aqueous $0.5 \mathrm{M} \mathrm{H}_{2} \mathrm{SeO}_{3}$ solution $\left(10 \mathrm{ml}\right.$, dissolved $\mathrm{SeO}_{2}$ ) to result in a clear solution. A mass of colourless platy crystals of (I), with a palepink tinge arising from a surface coating, grew as the water evaporated over the course of a few days.

\section{Crystal data}

$\begin{array}{ll}\mathrm{C}_{3} \mathrm{H}_{12} \mathrm{~N}_{2}{ }^{2+} \cdot \mathrm{SeO}_{3}{ }^{2-} \cdot \mathrm{H}_{2} \mathrm{O} & D_{x}=1.778 \mathrm{Mg} \mathrm{m}^{-3} \\ M_{r}=221.12 & \text { Mo } K \alpha \text { radiation } \\ \text { Monoclinic, } P 2 / n & \text { Cell parameters from } 1941 \\ a=11.5494(7) \AA & \quad \text { reflections } \\ b=6.1399(4) \AA & \theta=2.9-27.5^{\circ} \\ c=11.6601(6) \AA & \mu=4.51 \mathrm{~mm}^{-1} \\ \beta=92.213(3)^{\circ} & T=120(2) \mathrm{K} \\ V=826.23(8) \AA^{3} & \text { Plate, colourless } \\ Z=4 & 0.12 \times 0.10 \times 0.02 \mathrm{~mm}\end{array}$

Data collection

Nonius KappaCCD diffractometer $\omega$ and $\varphi$ scans

Absorption correction: multi-scan (SADABS; Bruker, 2003)

$T_{\min }=0.613, T_{\max }=0.915$

8108 measured reflections

1882 independent reflections

\section{Refinement}

Refinement on $F^{2}$

$R\left[F^{2}>2 \sigma\left(F^{2}\right)\right]=0.030$

$w R\left(F^{2}\right)=0.068$

$S=1.07$

1882 reflections

95 parameters

$\mathrm{H}$-atom parameters constrained

$$
\begin{aligned}
& 1617 \text { reflections with } I>2 \sigma(I) \\
& R_{\text {int }}=0.044 \\
& \theta_{\max }=27.5^{\circ} \\
& h=-12 \rightarrow 15 \\
& k=-7 \rightarrow 7 \\
& l=-15 \rightarrow 15
\end{aligned}
$$

Table 1

Selected geometric parameters $\left(\AA,^{\circ}\right)$.

\begin{tabular}{lrlr}
\hline $\mathrm{Se} 1-\mathrm{O} 1$ & $1.673(2)$ & $\mathrm{Se} 1-\mathrm{O} 2$ & $1.7052(18)$ \\
$\mathrm{Se} 1-\mathrm{O} 3$ & $1.6826(19)$ & & \\
$\mathrm{N} 1-\mathrm{C} 1-\mathrm{C} 2-\mathrm{N} 2$ & $55.2(3)$ & $\mathrm{C} 3-\mathrm{C} 1-\mathrm{C} 2-\mathrm{N} 2$ & $176.9(2)$ \\
\hline
\end{tabular}

Table 2

Hydrogen-bond geometry $\left(\AA,{ }^{\circ}\right)$.

\begin{tabular}{lllll}
\hline$D-\mathrm{H} \cdots A$ & $D-\mathrm{H}$ & $\mathrm{H} \cdots A$ & $D \cdots A$ & $D-\mathrm{H} \cdots A$ \\
\hline $\mathrm{N} 1-\mathrm{H} 1 \cdots \mathrm{O} 2^{\mathrm{i}}$ & 0.91 & 1.88 & $2.779(3)$ & 171 \\
$\mathrm{~N} 1-\mathrm{H} 2 \cdots \mathrm{O}{ }^{\mathrm{ii}}$ & 0.91 & 1.81 & $2.701(3)$ & 165 \\
$\mathrm{~N} 1-\mathrm{H} 3 \cdots \mathrm{O} 2$ & 0.91 & 2.00 & $2.835(3)$ & 152 \\
$\mathrm{~N} 2-\mathrm{H} 4 \cdots \mathrm{O} 4^{\mathrm{iii}}$ & 0.91 & 1.91 & $2.802(3)$ & 165 \\
$\mathrm{~N} 2-\mathrm{H} 5 \cdots \mathrm{O} 3$ & 0.91 & 1.87 & $2.777(3)$ & 173 \\
$\mathrm{~N} 2-\mathrm{H} 6 \cdots \mathrm{O} 2^{\mathrm{ii}}$ & 0.91 & 1.88 & $2.766(3)$ & 164 \\
$\mathrm{O} 4-\mathrm{H} 13 \cdots \mathrm{O} 3$ & 0.88 & 1.96 & $2.840(3)$ & 178 \\
$\mathrm{O} 4-\mathrm{H} 14 \cdots \mathrm{O} 1^{\text {iv }}$ & 0.76 & 2.04 & $2.747(3)$ & 157 \\
\hline Symmetry codes: & (i) $-x+1,-y+1,-z ;$ & (ii) & $-x+1,-y+2,-z ;$ & (iii) \\
$-x+\frac{3}{2}, y+1,-z+\frac{1}{2} ;$ (iv) $x, y-1, z$. & & &
\end{tabular}




\section{organic papers}

The non-standard $P 2 / n$ setting of the space group was chosen in preference to $P 2 / c$ to avoid a unit cell with a very obtuse $\beta$ angle of $133.6^{\circ}$. The water $\mathrm{H}$ atoms were found in a difference map and refined as riding in their as-found relative positions (Table 2). $\mathrm{H}$ atoms bonded to $\mathrm{C}$ and $\mathrm{N}$ atoms were placed in idealized positions $(\mathrm{C}-\mathrm{H}=0.98-1.00 \AA$ and $\mathrm{N}-\mathrm{H}=0.91 \AA)$ and refined as riding, allowing for free rotation of the rigid $-X \mathrm{H}_{3}(X=\mathrm{C} 3, \mathrm{~N} 1$ and $\mathrm{N} 2)$ groups. The constraint $U_{\text {iso }}(\mathrm{H})=1.2 U_{\text {eq }}($ carrier $)$ or $U_{\text {iso }}(\mathrm{H})=$ $1.2 U_{\text {eq }}$ (methyl carrier) was applied as appropriate.

Data collection: COLLECT (Nonius, 1999); cell refinement: $D E N Z O$ (Otwinowski \& Minor, 1997) and COLLECT; data reduction: DENZO and COLLECT; program(s) used to solve structure: SHELXS97 (Sheldrick, 1997); program(s) used to refine structure: SHELXL97 (Sheldrick, 1997); molecular graphics: ORTEP-3 (Farrugia, 1997); software used to prepare material for publication: SHELXL97.
We thank the EPSRC National Crystallography Service (University of Southampton, England) for the data collection.

\section{References}

Bruker (2003). SADABS. Bruker AXS Inc., Madison, Wisconsin, USA.

Demir, S., Yilmaz, V. T. \& Harrison, W. T. A. (2003). Acta Cryst. E59, o907o909.

Farrugia, L. J. (1997). J. App. Cryst. 30, 565.

Harrison, W. T. A. (2003). Acta Cryst. E59, o1267-o1269.

Lee, C. \& Harrison, W. T. A. (2003a). Acta Cryst. E59, m739-m741.

Lee, C. \& Harrison, W. T. A. (2003b). Acta Cryst. E59, m959-m960.

Lee, C. \& Harrison, W. T. A. (2003c). Acta Cryst. E59, m1151-m1153.

Nonius (1999). COLLECT. Nonius, Delft, The Netherlands.

Otwinowski, Z. \& Minor, W. (1997). Methods in Enzymology, Vol. 276, Macromolecular Crystallography, Part A, edited by C. W. Carter \& R. M. Sweet, pp. 307-326. London: Academic Press.

Ritchie, L. K. \& Harrison, W. T. A. (2003). Acta Cryst. E59, o1296-o1298.

Sheldrick, G. M. (1997). SHELXS97 and SHELXL97. University of Göttingen, Germany.

Wilkinson, H. S. \& Harrison, W. T. A. (2004). Acta Cryst. E60, m1359-m1361. 


\section{supporting information}

Acta Cryst. (2005). E61, o1538-o1540 [https://doi.org/10.1107/S160053680501278X]

\section{Propane-1,2-diaminium selenite monohydrate}

\section{Malcolm J. Todd and William T. A. Harrison}

Propane-1,2-diaminium selenite monohydrate

\section{Crystal data}

$\mathrm{C}_{3} \mathrm{H}_{12} \mathrm{~N}_{2}{ }^{2+} \cdot \mathrm{SeO}_{3}{ }^{2-} \cdot \mathrm{H}_{2} \mathrm{O}$

$M_{r}=221.12$

Monoclinic, $P 2 / n$

Hall symbol: $-\mathrm{P} 2 \mathrm{yac}$

$a=11.5494(7) \AA$

$b=6.1399(4) \AA$

$c=11.6601(6) \AA$

$\beta=92.213(3)^{\circ}$

$V=826.23(8) \AA^{3}$

$Z=4$

$F(000)=448$

$D_{\mathrm{x}}=1.778 \mathrm{Mg} \mathrm{m}^{-3}$

Mo $K \alpha$ radiation, $\lambda=0.71073 \AA$

Cell parameters from 1941 reflections

$\theta=2.9-27.5^{\circ}$

$\mu=4.51 \mathrm{~mm}^{-1}$

$T=120 \mathrm{~K}$

Plate, colourless

$0.12 \times 0.10 \times 0.02 \mathrm{~mm}$

\section{Data collection}

Nonius KappaCCD diffractometer

Radiation source: fine-focus sealed tube

Graphite monochromator

$\omega$ and $\varphi$ scans

Absorption correction: multi-scan

(SADABS; Bruker, 2003)

$T_{\min }=0.613, T_{\max }=0.915$

\section{Refinement}

Refinement on $F^{2}$

Least-squares matrix: full

$R\left[F^{2}>2 \sigma\left(F^{2}\right)\right]=0.030$

$w R\left(F^{2}\right)=0.068$

$S=1.07$

1882 reflections

95 parameters

0 restraints

Primary atom site location: structure-invariant direct methods

Secondary atom site location: difference Fourier map

8108 measured reflections

1882 independent reflections

1617 reflections with $I>2 \sigma(I)$

$R_{\text {int }}=0.044$

$\theta_{\max }=27.5^{\circ}, \theta_{\min }=3.3^{\circ}$

$h=-12 \rightarrow 15$

$k=-7 \rightarrow 7$

$l=-15 \rightarrow 15$

Hydrogen site location: difmap (O-H) and geom (C-N and N-H)

$\mathrm{H}$-atom parameters constrained

$w=1 /\left[\sigma^{2}\left(F_{\mathrm{o}}^{2}\right)+(0.0274 P)^{2}+0.5437 P\right]$

where $P=\left(F_{\mathrm{o}}{ }^{2}+2 F_{\mathrm{c}}{ }^{2}\right) / 3$

$(\Delta / \sigma)_{\max }<0.001$

$\Delta \rho_{\max }=0.98$ e $\AA^{-3}$

$\Delta \rho_{\min }=-0.51$ e $\AA^{-3}$

Extinction correction: SHELXL97, $\mathrm{Fc}^{*}=\mathrm{kFc}\left[1+0.001 \mathrm{xFc}^{2} \lambda^{3} / \sin (2 \theta)\right]^{-1 / 4}$

Extinction coefficient: 0.0026 (7) 


\section{Special details}

Geometry. All e.s.d.'s (except the e.s.d. in the dihedral angle between two 1.s. planes) are estimated using the full covariance matrix. The cell e.s.d.'s are taken into account individually in the estimation of e.s.d.'s in distances, angles and torsion angles; correlations between e.s.d.'s in cell parameters are only used when they are defined by crystal symmetry. An approximate (isotropic) treatment of cell e.s.d.'s is used for estimating e.s.d.'s involving l.s. planes.

Refinement. Refinement of $F^{2}$ against ALL reflections. The weighted $R$-factor $w R$ and goodness of fit $S$ are based on $F^{2}$, conventional $R$-factors $R$ are based on $F$, with $F$ set to zero for negative $F^{2}$. The threshold expression of $F^{2}>\sigma\left(F^{2}\right)$ is used only for calculating $R$-factors(gt) etc. and is not relevant to the choice of reflections for refinement. $R$-factors based on $F^{2}$ are statistically about twice as large as those based on $F$, and $R$ - factors based on ALL data will be even larger.

Fractional atomic coordinates and isotropic or equivalent isotropic displacement parameters $\left(\AA^{2}\right)$

\begin{tabular}{lllll}
\hline & $x$ & $y$ & $z$ & $U_{\text {iso }} * / U_{\text {eq }}$ \\
\hline Se1 & $0.44873(2)$ & $0.81651(4)$ & $0.21940(2)$ & $0.01546(12)$ \\
O1 & $0.43374(17)$ & $1.0875(3)$ & $0.22349(16)$ & $0.0253(5)$ \\
O2 & $0.42321(16)$ & $0.7560(3)$ & $0.07795(16)$ & $0.0204(4)$ \\
O3 & $0.59257(16)$ & $0.7750(3)$ & $0.23366(16)$ & $0.0209(4)$ \\
C1 & $0.7531(2)$ & $0.6926(4)$ & $0.0105(2)$ & $0.0180(6)$ \\
H7 & 0.7578 & 0.6212 & 0.0876 & $0.022^{*}$ \\
C2 & $0.7916(2)$ & $0.9266(4)$ & $0.0239(2)$ & $0.0169(5)$ \\
H8 & 0.8708 & 0.9299 & 0.0595 & $0.020^{*}$ \\
H9 & 0.7947 & 0.9943 & -0.0529 & $0.020^{*}$ \\
C3 & $0.8323(2)$ & $0.5718(5)$ & $-0.0684(2)$ & $0.0240(6)$ \\
H10 & 0.8072 & 0.4198 & -0.0755 & $0.036^{*}$ \\
H11 & 0.9120 & 0.5769 & -0.0364 & $0.036^{*}$ \\
H12 & 0.8290 & 0.6407 & -0.1443 & $0.036^{*}$ \\
N1 & $0.63165(19)$ & $0.6781(3)$ & $-0.03598(19)$ & $0.0169(5)$ \\
H1 & 0.6158 & 0.5383 & -0.0573 & $0.020^{*}$ \\
H2 & 0.6225 & 0.7671 & -0.0981 & $0.020^{*}$ \\
H3 & 0.5824 & 0.7199 & 0.0189 & $0.020^{*}$ \\
N2 & $0.71339(19)$ & $1.0567(4)$ & $0.09542(19)$ & $0.0180(5)$ \\
H4 & 0.7554 & 1.1595 & 0.1349 & $0.022^{*}$ \\
H5 & 0.6784 & 0.9673 & 0.1458 & $0.022^{*}$ \\
H6 & 0.6586 & 1.1229 & 0.0494 & $0.022^{*}$ \\
O4 & $0.6226(18)$ & $0.3308(3)$ & $0.29602(19)$ & $0.0283(5)$ \\
H13 & 0.6129 & 0.4666 & 0.2746 & $0.034^{*}$ \\
H14 & 0.5633 & 0.2771 & 0.2923 & $0.034^{*}$ \\
& & & & \\
\hline
\end{tabular}

Atomic displacement parameters $\left(\AA^{2}\right)$

\begin{tabular}{lllllll}
\hline & $U^{11}$ & $U^{22}$ & $U^{33}$ & $U^{12}$ & $U^{13}$ & $U^{23}$ \\
\hline Se1 & $0.01747(18)$ & $0.01530(16)$ & $0.01357(16)$ & $-0.00069(11)$ & $0.00008(11)$ & $0.00103(10)$ \\
O1 & $0.0291(11)$ & $0.0169(10)$ & $0.0293(11)$ & $0.0055(9)$ & $-0.0071(9)$ & $-0.0067(9)$ \\
O2 & $0.0239(11)$ & $0.0173(9)$ & $0.0194(10)$ & $0.0011(8)$ & $-0.0077(8)$ & $-0.0037(8)$ \\
O3 & $0.0166(10)$ & $0.0209(10)$ & $0.0248(10)$ & $-0.0008(8)$ & $-0.0037(8)$ & $0.0036(8)$ \\
C1 & $0.0180(14)$ & $0.0158(13)$ & $0.0203(14)$ & $0.0017(11)$ & $-0.0001(11)$ & $-0.0008(11)$ \\
C2 & $0.0144(13)$ & $0.0173(13)$ & $0.0191(13)$ & $-0.0005(11)$ & $-0.0008(10)$ & $-0.0007(11)$
\end{tabular}




\begin{tabular}{lllllll}
$\mathrm{C} 3$ & $0.0198(15)$ & $0.0236(15)$ & $0.0288(15)$ & $0.0048(12)$ & $0.0049(12)$ & $0.0005(13)$ \\
$\mathrm{N} 1$ & $0.0175(12)$ & $0.0137(11)$ & $0.0195(12)$ & $0.0007(9)$ & $-0.0002(9)$ & $-0.0049(9)$ \\
$\mathrm{N} 2$ & $0.0178(12)$ & $0.0142(11)$ & $0.0216(11)$ & $-0.0006(9)$ & $-0.0060(9)$ & $0.0000(9)$ \\
$\mathrm{O} 4$ & $0.0199(11)$ & $0.0156(10)$ & $0.0482(13)$ & $0.0024(8)$ & $-0.0131(10)$ & $0.0040(9)$ \\
\hline
\end{tabular}

Geometric parameters $\left(\AA,{ }^{\circ}\right)$

\begin{tabular}{|c|c|c|c|}
\hline $\mathrm{Se} 1-\mathrm{O} 1$ & $1.673(2)$ & $\mathrm{C} 3-\mathrm{H} 11$ & 0.9800 \\
\hline $\mathrm{Se} 1-\mathrm{O} 3$ & $1.6826(19)$ & $\mathrm{C} 3-\mathrm{H} 12$ & 0.9800 \\
\hline $\mathrm{Se} 1-\mathrm{O} 2$ & $1.7052(18)$ & $\mathrm{N} 1-\mathrm{H} 1$ & 0.9100 \\
\hline $\mathrm{C} 1-\mathrm{N} 1$ & $1.487(3)$ & $\mathrm{N} 1-\mathrm{H} 2$ & 0.9100 \\
\hline $\mathrm{C} 1-\mathrm{C} 2$ & $1.510(4)$ & $\mathrm{N} 1-\mathrm{H} 3$ & 0.9100 \\
\hline $\mathrm{C} 1-\mathrm{C} 3$ & $1.516(4)$ & $\mathrm{N} 2-\mathrm{H} 4$ & 0.9100 \\
\hline $\mathrm{C} 1-\mathrm{H} 7$ & 1.0000 & $\mathrm{~N} 2-\mathrm{H} 5$ & 0.9100 \\
\hline $\mathrm{C} 2-\mathrm{N} 2$ & $1.485(3)$ & N2-H6 & 0.9100 \\
\hline $\mathrm{C} 2-\mathrm{H} 8$ & 0.9900 & $\mathrm{O} 4-\mathrm{H} 13$ & 0.8761 \\
\hline $\mathrm{C} 2-\mathrm{H} 9$ & 0.9900 & $\mathrm{O} 4-\mathrm{H} 14$ & 0.7566 \\
\hline $\mathrm{C} 3-\mathrm{H} 10$ & 0.9800 & & \\
\hline $\mathrm{O} 1-\mathrm{Se} 1-\mathrm{O} 3$ & $104.52(9)$ & $\mathrm{H} 10-\mathrm{C} 3-\mathrm{H} 11$ & 109.5 \\
\hline $\mathrm{O} 1-\mathrm{Se} 1-\mathrm{O} 2$ & $103.29(9)$ & $\mathrm{C} 1-\mathrm{C} 3-\mathrm{H} 12$ & 109.5 \\
\hline $\mathrm{O} 3-\mathrm{Se} 1-\mathrm{O} 2$ & $101.28(9)$ & $\mathrm{H} 10-\mathrm{C} 3-\mathrm{H} 12$ & 109.5 \\
\hline $\mathrm{N} 1-\mathrm{C} 1-\mathrm{C} 2$ & $111.4(2)$ & $\mathrm{H} 11-\mathrm{C} 3-\mathrm{H} 12$ & 109.5 \\
\hline $\mathrm{N} 1-\mathrm{C} 1-\mathrm{C} 3$ & $109.4(2)$ & $\mathrm{C} 1-\mathrm{N} 1-\mathrm{H} 1$ & 109.5 \\
\hline $\mathrm{C} 2-\mathrm{C} 1-\mathrm{C} 3$ & $110.3(2)$ & $\mathrm{C} 1-\mathrm{N} 1-\mathrm{H} 2$ & 109.5 \\
\hline $\mathrm{N} 1-\mathrm{C} 1-\mathrm{H} 7$ & 108.6 & $\mathrm{H} 1-\mathrm{N} 1-\mathrm{H} 2$ & 109.5 \\
\hline $\mathrm{C} 2-\mathrm{C} 1-\mathrm{H} 7$ & 108.6 & $\mathrm{C} 1-\mathrm{N} 1-\mathrm{H} 3$ & 109.5 \\
\hline $\mathrm{C} 3-\mathrm{C} 1-\mathrm{H} 7$ & 108.6 & $\mathrm{H} 1-\mathrm{N} 1-\mathrm{H} 3$ & 109.5 \\
\hline $\mathrm{N} 2-\mathrm{C} 2-\mathrm{C} 1$ & $112.8(2)$ & $\mathrm{H} 2-\mathrm{N} 1-\mathrm{H} 3$ & 109.5 \\
\hline $\mathrm{N} 2-\mathrm{C} 2-\mathrm{H} 8$ & 109.0 & $\mathrm{C} 2-\mathrm{N} 2-\mathrm{H} 4$ & 109.5 \\
\hline $\mathrm{C} 1-\mathrm{C} 2-\mathrm{H} 8$ & 109.0 & $\mathrm{C} 2-\mathrm{N} 2-\mathrm{H} 5$ & 109.5 \\
\hline $\mathrm{N} 2-\mathrm{C} 2-\mathrm{H} 9$ & 109.0 & $\mathrm{H} 4-\mathrm{N} 2-\mathrm{H} 5$ & 109.5 \\
\hline $\mathrm{C} 1-\mathrm{C} 2-\mathrm{H} 9$ & 109.0 & $\mathrm{C} 2-\mathrm{N} 2-\mathrm{H} 6$ & 109.5 \\
\hline $\mathrm{H} 8-\mathrm{C} 2-\mathrm{H} 9$ & 107.8 & $\mathrm{H} 4-\mathrm{N} 2-\mathrm{H} 6$ & 109.5 \\
\hline $\mathrm{C} 1-\mathrm{C} 3-\mathrm{H} 10$ & 109.5 & $\mathrm{H} 5-\mathrm{N} 2-\mathrm{H} 6$ & 109.5 \\
\hline $\mathrm{C} 1-\mathrm{C} 3-\mathrm{H} 11$ & 109.5 & $\mathrm{H} 13-\mathrm{O} 4-\mathrm{H} 14$ & 107.3 \\
\hline $\mathrm{N} 1-\mathrm{C} 1-\mathrm{C} 2-\mathrm{N} 2$ & $55.2(3)$ & $\mathrm{C} 3-\mathrm{C} 1-\mathrm{C} 2-\mathrm{N} 2$ & $176.9(2)$ \\
\hline
\end{tabular}

Hydrogen-bond geometry $\left(\AA,{ }^{\circ}\right)$

\begin{tabular}{lllll}
\hline$D-\mathrm{H} \cdots A$ & $D-\mathrm{H}$ & $\mathrm{H} \cdots A$ & $D \cdots A$ & $D-\mathrm{H} \cdots A$ \\
\hline $\mathrm{N} 1-\mathrm{H} 1 \cdots \mathrm{O} 2^{\mathrm{i}}$ & 0.91 & 1.88 & $2.779(3)$ & 171 \\
$\mathrm{~N} 1-\mathrm{H} 2 \cdots \mathrm{O} 1^{\mathrm{ii}}$ & 0.91 & 1.81 & $2.701(3)$ & 165 \\
$\mathrm{~N} 1-\mathrm{H} 3 \cdots \mathrm{O} 2$ & 0.91 & 2.00 & $2.835(3)$ & 152 \\
$\mathrm{~N} 2-\mathrm{H} 4 \cdots \mathrm{O} 4^{\mathrm{iii}}$ & 0.91 & 1.91 & $2.802(3)$ & 165 \\
$\mathrm{~N} 2-\mathrm{H} 5 \cdots \mathrm{O} 3$ & 0.91 & 1.87 & $2.777(3)$ & 173 \\
$\mathrm{~N} 2-\mathrm{H} 6 \cdots \mathrm{O} 2^{\text {ii }}$ & 0.91 & 1.88 & $2.766(3)$ & 164
\end{tabular}


supporting information

$\mathrm{O} 4-\mathrm{H} 13 \cdots \mathrm{O} 3$

0.88

1.96

$2.840(3)$

178

$\mathrm{O} 4-\mathrm{H} 14 \cdots \mathrm{O} 1^{\text {iv }}$

2.04

2.747 (3)

157

Symmetry codes: (i) $-x+1,-y+1,-z$; (ii) $-x+1,-y+2,-z$; (iii) $-x+3 / 2, y+1,-z+1 / 2$; (iv) $x, y-1, z$. 